UVX 2008 (2009) 85-91

(C) EDP Sciences, 2009

DOI: $10.1051 / \mathrm{uvx} / 2009014$

\title{
The SCSS test accelerator Free-Electron Laser seeded by harmonics produced in gas
}

\author{
G. Lambert ${ }^{1}$, T. Hara ${ }^{2}$, T. Tanikawa ${ }^{3}$, D. Garzella ${ }^{4}$, B. Carré ${ }^{4}$, \\ T. Ishikawa ${ }^{2}$, H. Kitamura ${ }^{2}$, T. Shintake ${ }^{2}$, K. Tahara ${ }^{2}$, Y. Tanaka ${ }^{2}$, \\ M. Yabashi ${ }^{2}$, M. Bougeard ${ }^{4}$, M. Labat ${ }^{4}$, H. Merdji ${ }^{4}$, P. Salières ${ }^{4}$, \\ O. Gobert ${ }^{4}$, O. Chubar ${ }^{5}$ and M.E. Couprie ${ }^{5}$ \\ ${ }^{1}$ LOA ENSTA Palaiseau, France \\ 2SPring-8/RIKEN, Hyogo, Japan \\ ${ }^{3}$ UVSOR Facility, Okazaki, Japan \\ ${ }^{4}$ CEA Saclay, DSMISPAM, France \\ ${ }^{5}$ Synchrotron Soleil, Saint-Aubin, France
}

\begin{abstract}
Today, single-pass Free-Electron Lasers (FEL) produce a highly bright radiation, the Self Amplified Spontaneous Emission (SASE), which spectral and temporal profiles are composed of a series of spikes. We demonstrate here the strong and coherent amplification of the $5^{\text {th }}$ harmonic of a Ti: Sa laser $(800 \mathrm{~nm}, 10 \mathrm{~Hz}, 100 \mathrm{fs}$ ) generated in a gas cell, i.e. $160 \mathrm{~nm}$, by the SCSS (SPring-8 Compact SASE Source, Japan) Test Accelerator FEL. This is obtained by overlapping transversally, spectrally and temporally the external harmonic source in the in-vacuum undulator with the electron beam $(150 \mathrm{MeV}, 10 \mathrm{~Hz}, 1 \mathrm{ps})$. With only one undulator section, the $160 \mathrm{~nm}$ seeded emission achieves three orders of magnitude higher intensity than the unseeded one, and presents a quasi perfect Gaussian shape in the spectral distribution. With two undulator sections, the seeded FEL spectrum reveals first effects of saturation. Those spectacular phenomena are associated to the generation of intense and coherent Non Linear Harmonics (NLH) at $54 \mathrm{~nm}$ and $32 \mathrm{~nm}$. Finally, in view of the low seed level required, such amplification associated to NLH schemes would allow the generation of fully coherent soft X-ray radiations down to the water window.
\end{abstract}

\section{INTRODUCTION}

Nowadays, most of the new FEL sources are dedicated to the Self Amplified Spontaneous Emission (SASE) $[1,2]$, which provides with a very high brightness emission at short wavelength but with limited temporal coherence. In 2000, a seeded FEL at $1.06 \mu$ m combined to the generation of coherent harmonics has been demonstrated experimentally [3], establishing that the seeded beam gave its full coherence property to the emitted radiation and allowed to decrease the saturation length in a more compact source. Also, injection of a single-pass FEL by the $3^{\text {rd }}$ laser harmonic of a Ti:Sapphire laser from crystals ( $266 \mathrm{~nm}, 4 \mu \mathrm{J}$, $5 \mathrm{ps}$ FWHM) led to large amplification [4]. Seeding a FEL with high-order laser harmonics generated in gas (HHG), which present high degrees of spatial and temporal coherence [5], offers an extension to short wavelength. Indeed, HHG sources now exist down to the water window [6,7]. Consequently, seeding a FEL [8-11] becomes relevant for generating intense and fully coherent short wavelength radiation. Even these last years, a few FEL facilities based on the SASE emission have decided to implement the HHG seeding concept, like the SCSS (SPring-8 Compact SASE Source, Japan) Test Accelerator [8-10], SPARC (Sorgente Pulsata e Amplificata di Radiazione Coerente, Italy) [11] and FLASH (Free-electron LASer in Hamburg, Germany) [2].

\section{GENERAL PRESENTATION}

The seeding experiment ([10], Fig. 1) has been carried out on the FEL of the SCSS Test Accelerator $[12,13]$ at $160 \mathrm{~nm}$, which corresponds to the $5^{\text {th }}$ harmonic of a Ti:Sa laser, with a $150 \mathrm{MeV}$ 


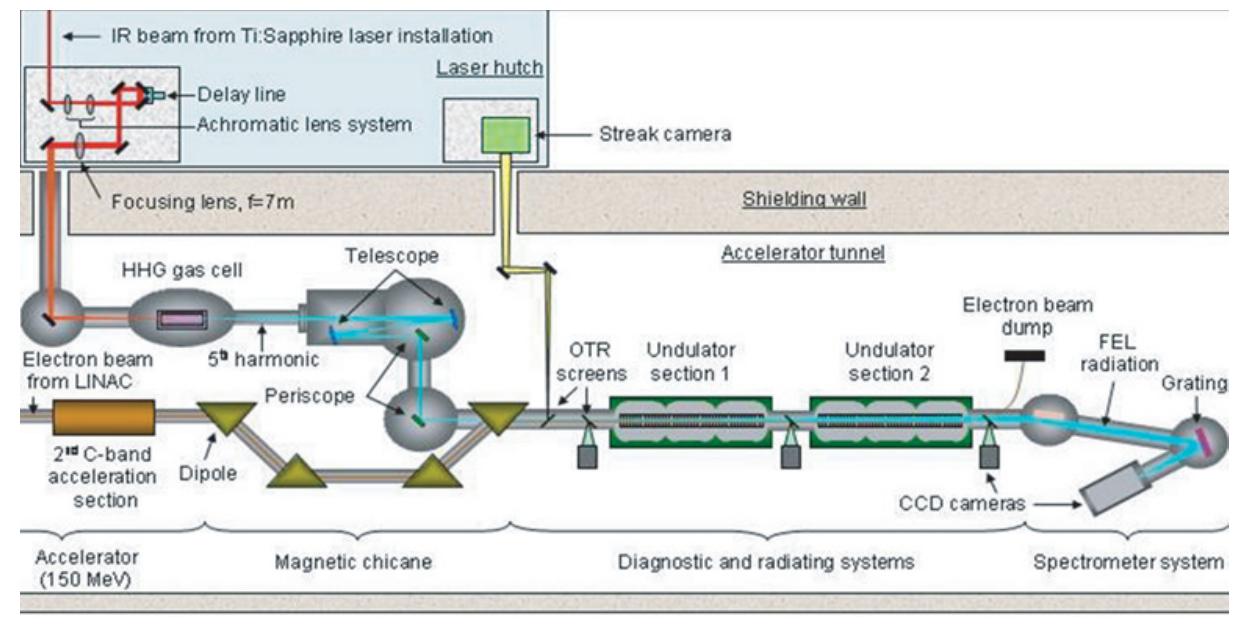

Figure 1. General layout of the seeding experiment with harmonics generated in a gas cell.

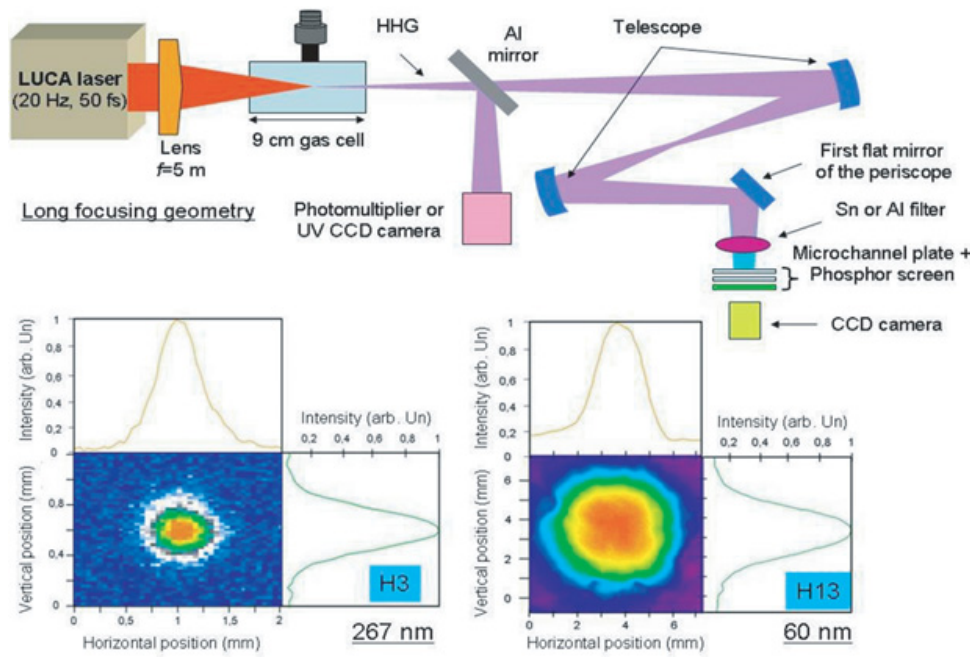

Figure 2. Scheme of the HHG system developed in CEA Saclay for testing the generation of law and high harmonic orders (respectively the $3^{\text {rd }}$ one at $267 \mathrm{~nm}$ and $13^{\text {th }}$ one at $60 \mathrm{~nm}$ ) in a long focussing geometry.

electron beam. This facility is mainly based on a thermionic cathode electron gun ( $1 \mathrm{nC})$, a C-band LINAC $(5712 \mathrm{MHz})$ and an in-vacuum undulator $(15 \mathrm{~mm}$ of period, two $4.5 \mathrm{~m}$ long sections). In June 2006, the first lasing has been observed at $49 \mathrm{~nm} \mathrm{[14]} \mathrm{with} \mathrm{a} 250 \mathrm{MeV}$ ebeam and the full saturation has been reached in 2007 [15]. From the HHG part, the experiment has been designed and tested in France in 2005 (Fig. 2) and finally implemented (Fig. 1) inside the accelerator tunnel in Japan in 2006.

The harmonic generation in gas results from the strong non linear polarization induced on the rare gases atoms, such as $\mathrm{Ar}, \mathrm{Xe}, \mathrm{Ne}$ and $\mathrm{He}$, by the focusing of an intense electromagnetic field of a "pump" laser. The laser system, in our case, is based on the Chirped Pulsed Amplification (CPA) Ti: Sa technology, and is mainly composed of a Tsunami mode-locked oscillator, a Spitfire regenerative 


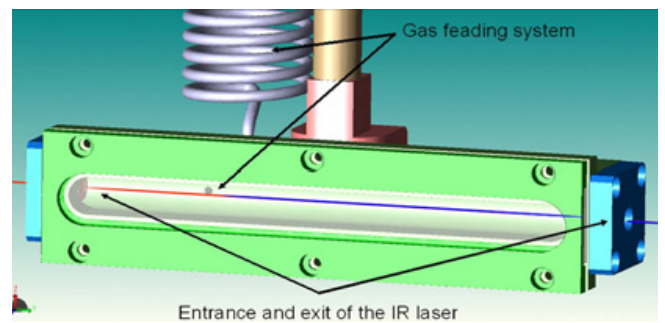

Figure 3. Gas cell system for producing the HHG light.

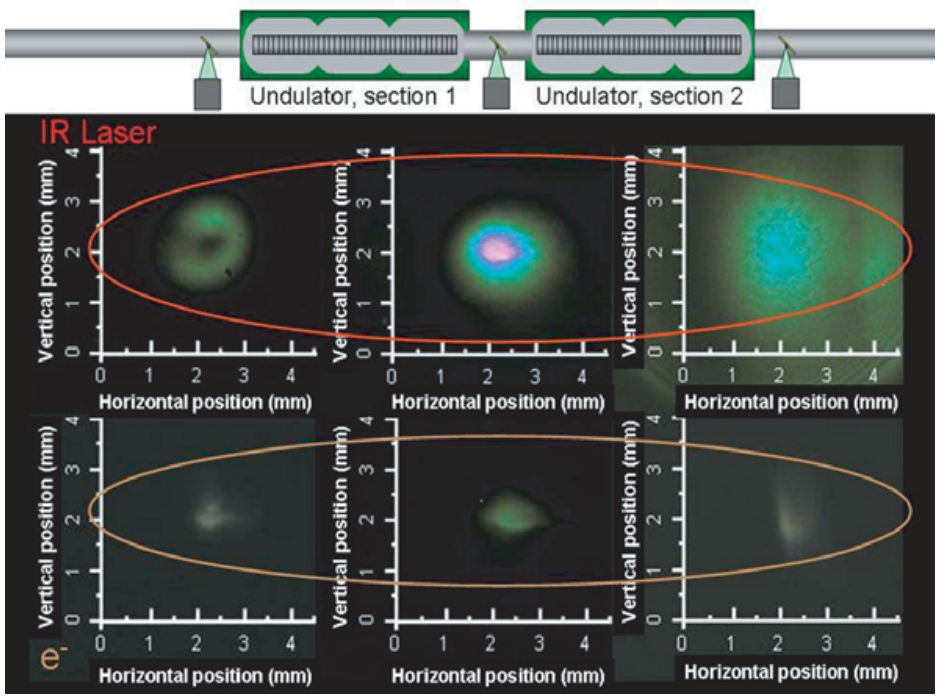

Figure 4. CCD pictures of the IR and electron beams OTR emissions for aligning the harmonics on the undulator emission axis.

amplifier and a multipass amplifier. It delivers a high energy IR laser beam $(<50 \mathrm{~mJ}, 10 \mathrm{~Hz}, 100 \mathrm{fs})$, which is focalized by a lens into a cell (Fig. 3) filled with Xe, which is a well-adapted gas for the generation of the $160 \mathrm{~nm}$ radiation.

As the Ti: Sa laser system is installed outside the tunnel of the accelerator and in order to pass through the shielding wall ( $2.3 \mathrm{~m}$ of height for radiation safety reasons), the IR beam is propagated on two IR periscope systems helping for the alignment in the gas cell, located in the first chamber of the harmonic generation experiment. The laser passes through the cell and is aligned by means of two pinholes (Tantalus plates) of $1 \mathrm{~mm}$ of diameter, which create a constant leak of gas (Fig. 3).

The second chamber contains the VUV telescope and periscope systems. They allow the generated $5^{\text {th }}$ harmonic to be efficiently propagated, and refocalized into the first undulator section, taking advantage of the magnetic chicane, in order to adapt its divergence as closed as possible to the electron beam one. Also, both beams have to be precisely transversally overlapped (Fig. 4) all along the entire undulator and synchronized with subfemtosecond precision.

\section{GENERATION OF HIGH ENERGY SEED}

For optimizing the harmonic generation process, the IR laser beam parameters have to be adjusted. Using an afocal system, the diameter of the multipass amplifier beam is enhanced by a factor of 5 , for 
decreasing the power density on the propagation mirrors and in opposite to have a shorter waist in the gas cell which gives a higher power density for the generation process. A final iris allows the geometry and the density profile of the beam to be finely changed. With those systems, the energy is reduced roughly to $20 \mathrm{~mJ}$ in a $20 \mathrm{~mm}$ full diameter. The polarization of the IR beam, which is originally horizontal, is switched twice during the transport over the concrete wall to be retransformed into the horizontal one. Indeed, as the harmonic beam presents the same polarization as the IR beam, it enters with a horizontal electric field inside the first undulator section, especially designed for amplification in this polarization. Finally, the gas pressure, entering the gas cell, is regulated by changing the aperture of an up-stream micro-leak valve, placed outside the accelerator. The gas pressure evolution can be followed by reading a calibrated pressure detector directly located after the micro-leak valve.

\section{OPTICAL ALIGNMENT}

It is needed that the seed radiation be co-propagated with the electron beam for having a proper overlap inside the first undulator section. The alignment is performed on OTR (Optical Transition Radiation) screens, which are disposed on the electron beam way at entrance and exit of each undulator section. Actually, as the harmonics are collinear to the IR laser, which is intense and reflected by the OTR screens, one should align the IR laser on the ebeam. By looking at the corresponded CCD camera screens (Fig. 4) and adjusting the angles of the two flat mirrors of the VUV periscope, the two beams are superposed. Finally, in order to focus the harmonic beam at various positions inside the first undulator section, a translation stage has been added below the second spherical mirror of the VUV telescope.

\section{SYNCHRONIZATION}

The synchronization between the electron beam and the seed pulse is achieved by locking the Ti:Sapphire oscillator to the highly stable $240 \mathrm{MHz}$ clock of the accelerator. The timing is then adjusted with a few ps resolution using a femtosecond streak camera (Hamamatsu Photonics FESCA-200C6138), on which the IR laser light and the OTR emission from the electron beam can be observed. Finally, a motorized fine optical delay line, installed on the IR laser path, allows reaching fs level temporal overlap between the IR laser, and so the harmonics, and the electron beam.

\section{RESULTS}

The amplification of the seed occurs through the two undulator sections, separated by a $1.5 \mathrm{~m}$ length drift space, and the final radiation is observed on the CCD camera of a dispersive spectrometer. As the photon beam is dispersed horizontally by the grating, the CCD camera reveals pictures (Fig. 5), in which the vertical axis is the vertical position of the beam and the horizontal axis is the wavelength. When the $160 \mathrm{~nm}$ seed is well aligned and spectrally matched with the undulator emission axis, the FEL seeded radiation can be highly amplified on that axis. For instance, with only one undulator section set in resonance and with a seed of $0.53 \mathrm{~nJ}$ energy per pulse ( $\approx 40 \mathrm{fs} F W \mathrm{FH})$, the energy reached by the FEL is estimated to be about $250 \mathrm{~nJ}$. Moreover, the spectral emission presents regular quasi perfect Gaussian shape pulse to pulse, slightly red-shifted compared to the normal radiation, which is an effect due to the additional exchange of energy with the ebeam. The spatial shape is similar to the unseeded emission, meaning that the good spatial coherence of the FEL is at least kept.

Once both undulator segments are set in resonance with the seed wavelength, the spectral distribution exhibits a sideband structure (Fig. 6), probably indicating an over-bunching of the electrons performing synchrotron oscillations at saturation [16]. The spectral red-shift approximately reaches twice larger 


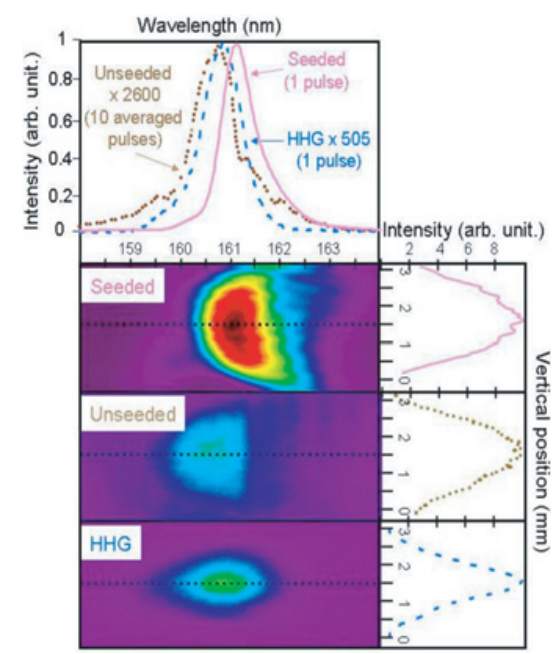

Figure 5. Comparison between the FEL seeded emission, the unseeded emission and the HHG seed at the fundamental wavelength $(160 \mathrm{~nm})$. The 1D-spatial (vertical) and spectral distributions are mapped on the CCD camera of a spectrometer; spatial (right) and spectral (up) profiles are plotted at maximum intensity. The lines correspond to the seeded (single shot), and unseeded (averaged on 10 shots) emissions, and the HHG seed (single shot). The seed pulse energy was $0.53 \mathrm{~nJ}$ and only the first undulator section was used for amplifying the HHG pulse.

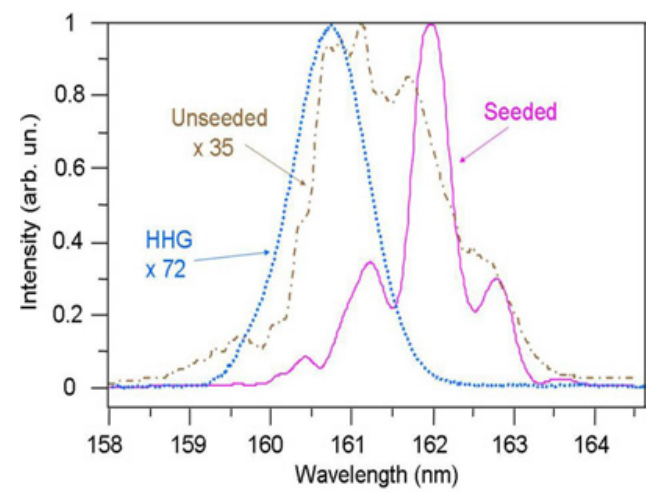

Figure 6. Spectra of the FEL fundamental single shot emission at $160 \mathrm{~nm}$ using the two undulator sections and obtained with a $4.3 \mathrm{~nJ}$ seed. The FEL gain is smaller compared with the measurements of Fig. 5, because of lower electron beam brightness, transverse misalignment and spectral detuning.

values $(\approx 2.5)$ compared with the single undulator section case, demonstrating additional energy transfer from the electron beam to the FEL field.

This amplification is followed by the generation of coherent FEL non linear harmonics (NLH), and more particularly the $3^{\text {rd }}$ and $5^{\text {th }}$ ones (Fig. 7), respectively at $54 \mathrm{~nm}(\approx 300 \mathrm{pJ}, \approx 40 \mathrm{fs}$ FWHM) and $32 \mathrm{~nm}(\approx 10 \mathrm{pJ}, \approx 40 \mathrm{fs}$ FWHM $)$. The NLH show similar spectral behaviors than the fundamental. Taking into account the pulse duration difference between the unseeded emission $(\approx 1 \mathrm{ps})$ and the seeded one (close to the seed one), for the fundamental and also for NLH (slightly lower than the one of the fundamental), the measured spectral widths indicate that the temporal coherence is largely improved with the seed injection. 

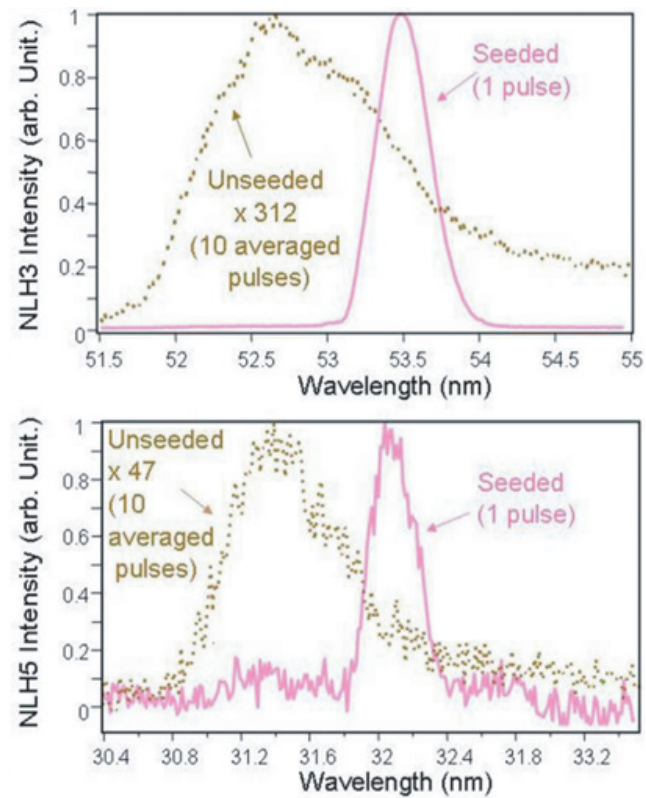

Figure 7. Spectra of the $3^{\text {rd }}$ and $5^{\text {th }}$ NLH of the $160 \mathrm{~nm}$ radiation. The seed pulse energy was $0.53 \mathrm{~nJ}$ and only the first undulator section was used for amplifying the HHG pulse.

\section{CONCLUSIONS}

Seeding a FEL with HHG radiations offers a real opportunity to spread the spectral range of the fully coherent FEL towards short wavelengths. In fact, these days, some table-top laser installations of harmonic generation produce radiations close to the water window and with notable peak powers. Also, in the view of the weak injection level required here $(<0.5 \mathrm{~nJ})$ and the strong factor of amplification attained, it is reasonable to imagine to realize in a short time a FEL, at a relatively low energy, allowing generating intense and totally coherent radiations at a nanometer scale wavelength. Besides, the generation system already allows to generate harmonic in the plateau region, i.e. from $70 \mathrm{~nm}$ to $30 \mathrm{~nm}$, and with a level of comparable energy to the one used here. Also, an immediate extension of this scheme to $60 \mathrm{~nm}$ is foreseen for 2008-2009.

\section{References}

[1] R. Bonifacio et al., Opt. Commun. 50 (6), (1984) 373-378.

[2] V. Ayvazyan et al., Eur. Phys. J. D. 37, (2006) 297-303.

[3] L. H. Yu et al., Science 289, (2000) 932-934.

[4] T. Shaftan et al., NIMA 507, (2003) 15-18.

[5] P. Salieres et al., Phys. Rev. Lett. 74 (19), (1995) 3776-3779.

[6] E. Takahashi et al., Appl. Phys. Lett. 84 (01), (2004) 4-6.

[7] J. Seres et al., Nature 433, (2005) 596.

[8] D. Garzella et al., NIMA 528, (2004) 502-505.

[9] G. Lambert et al., Proceedings of the 2006 EPAC conference (JACOW), (2006) 44-47.

[10] G. Lambert et al., Nature Physics 889 (4), (2008) 296-300.

[11] L. Giannessi et al., Proceedings of the 2006 EPAC conference (JACOW), (2006) 95-98.

[12] K. Togawa et al., Phys. Rev. ST Accel. Beams 10, 020703 (10), (2007). 
[13] H. Tanaka et al., Proceedings of the 2006 FEL conference (JACOW), (2006) 769-776.

[14] T. Shintake et al., Proceedings of the 2006 EPAC conference (JACOW), (2006) 2741-2743.

[15] T. Shintake et al., Nature Photonics 2 (9), (2008) 555-559.

[16] Z. Huang, and K. -J Kim., NIMA 483, (2002) 504-509. 